\title{
HACIA EL BUEN COMER Y LA SALUD DESDE LA EDUCACIÓN
}

\section{RESUMEN}

La pandemia del COVID-19 cambió nuestro estilo de vida, las palabras "distanciamiento social" impactaron las celebraciones, reuniones, reencuentros, paseos y con ello, nuestro gozo del comer en compañía... para después, con la esperanza del reencuentro. Viejos lineamientos de cuidado de salud individual retomaron actualidad y un escenario de incertidumbre cubrió nuestro día a día. Los expertos cubrían los noticieros con información científica de las posibles causas y consecuencias de la nueva enfermedad, y algunos no expertos daban consejos sobre un sin número de productos y extrañas mezclas sobre qué es bueno para evitar el enfermarse o disminuir el riego de morir.

Lo destacable es que, dentro de esa inmensa masa de información aparece con frecuencia la alimentación: los que no se alimentan bien son más susceptibles a contraer la enfermedad, aquellos con buenos hábitos de consumo son menos vulnerables. Adquirir comportamientos saludables forma parte de la educación que se imparte tanto en el hogar como desde las instituciones educativas o por acciones educativas en la Promoción de la Salud. El contexto de las posibilidades de la Educación Alimentaria Nutricional en el Perú se analiza en el presente artículo.

\section{PALABRAS CLAVE}

Alimentación, enfermedad, comportamiento, cuidado, saludable.

\section{ABSTRACT}

COVID-19 has changed our lifestyle. The words "social distancing" had impacted in celebrations, meetings, reunions, walking and thus, our joy of eating together... and then, with the hope of the reunion. The old guidelines regarding individual health care returned to be used and state of uncertainty covered our lives. The experts have covered the newscasts with science information of the possibly causes and consequences of COVID 19, and some no experts shared advices about an endless number of products and strange mixes about what is good to avoid getting sick or reducing the death risk.

The remarkable of all this information is that the word "food" appear frequently. People who don't have a good food are more susceptible to get sick. On the other hand; people who have good habits food are less vulnerable. To get healthy behaviors take part in the education which it is given both in home as from the educational institutions or educational actions in the Health Promotion. The context of the possibilities of the Nutritional Food Education in Peru is analyzed in the present article.

KEYWORDS

Food, disease, behavior, care, healthy.

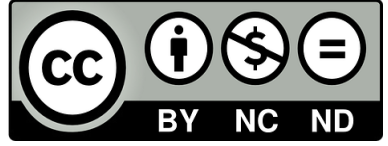

El uso que se haga de este artículo deberá incluir: Autor / Título original de la publicación / ISSN

\section{THE IMPACT OF AN EDUCATION ON A HEALTHY NUTRITION}

\author{
Yadira Rosa Jiménez Arrunátegui \\ ORCID: 0000-001-5509-8568 \\ yjimenez@unife.edu.pe
}

\section{INTRODUCCIÓN}

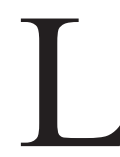

a Educación Alimentaria Nutricional

(EAN) forma parte de las estrategias

de organismos internacionales para hacer frente a problemas de malnutrición por carencia o por exceso y se incluyen en la agenda gubernamental de los países, en el marco de políticas públicas de salud, protección y bienestar infantil, bajo el contexto de la promoción de la salud y de la Seguridad Alimentaria y Nutricional (SAN), (Brasil. Ministerio de Desarrollo Social y Combate al Hambre, 2012, p. 13).

En el conjunto de los organismos internacionales, la Organización de las Naciones Unidas para la Agricultura y la Alimentación-FAO destaca el rol de la Educación Alimentaria Nutricional poniendo como centro los alimentos de la dieta, en cuanto son la base del crecimiento, desarrollo y capacidades de las personas, señalando que la información, la comunicación y la educación (ICEAN) van de la mano con la cultura alimentaria (Fondo de las Naciones Unidas para la Agricultura y la Alimentación, s/f, p. 4).

Con este mismo gran propósito, surge la iniciativa, América Latina y el Caribe sin hambre, que moviliza voluntades de muchas personas desde sus respectivas áreas profesionales y de conocimiento. Así prestigiosos chefs internacionales, cocineros, y críticos gastronómicos comprometen esfuerzos y recursos a través de programas educativos que 
buscan mostrar formas prácticas y sencillas de tener una dieta variada y rica, para animar a la población en general a preparar los alimentos base de la alimentación en la región con el propósito de mejorar la nutrición de los más vulnerables (La iniciativa América Latina y Caribe sin hambre, http://www.ialcsh.org/). La campaña Chefs contra el Hambre, tiene ya tres recetarios de carácter internacional dedicados a alimentos emblemáticos de la dieta latinoamericana, como son la papa, las legumbres y el maíz y el cuarto recetario, se centra en los frutos del mar: algas, mariscos y pescados (Chefs contra el Hambre, http://www.fao.org/).

En el Perú, la puesta en marcha de la estrategia mencionada desde la acción pública, contempla los diversos sectores vinculados al proceso de producción, distribución, abastecimiento $\mathrm{y}$ consumo de alimentos priorizando la atención de la población vulnerable. Los aspectos culturales de la alimentación se han incorporado para hacer de la Educación Alimentaria Nutricional, el centro de la prevención y control de los problemas alimentarios y nutricionales contemporáneos.

La Ley de la Alimentación Saludable abre opciones de cambio en la prevención $\mathrm{y}$ promoción de la salud, desde su mejor aliado, el sector educación para informar y comunicar, a partir de la cultura alimentaria, con intervenciones que mejoren la alimentación y nutrición de los escolares y contribuir así a su formación integral, acercando el conocimiento sobre los alimentos para una decisión informada sobre que comprar en los quioscos escolares o que consumir al orientar en la preparación de las loncheras escolares.

Las posibilidades de la Educación Alimentaria Nutricional en el Perú, se orientan a hacer accesible el conocimiento científico de la temática de la alimentación y nutrición disponible, para su uso en el contexto de la educación en instituciones públicas y privadas.

\section{Los alimentos nuestros de cada día}

Sin entrar en precisiones históricas sobre el origen de la agricultura, es significativo señalar que solo muy pocos pueblos cultos de Eurasia y África han alcanzado a producir un número alto de plantas alimenticias, como lo hizo el Perú prehispánico.

Se llega a identificar 44 tipos de plantas comestibles (H. Horkheimer, 2004, p. 95), las cuales, rescatando la tradición milenaria del buen comer, están en nuestra canasta de consumo familiar con los mismos nombres con los cuales eran conocidos por los españoles: maíz, yuca, guayaba, camote, maguey, caihua, maní, chirimoya o de nuestra lengua, el quechua: achira, kañawa/kañagua/qañigua; kiwicha/ Achita; tarwui (chochos, lupinos en castellano); oqa; mashwa/añu/isaña; r'qacha (arracacha, quichualización de la voz); llac'on (yacón, castellanización de su nombre) (F. Herrera, 1941, p. 11).

El maíz, "la sagrada planta" que nos lleva a evocar los tamales con maní de Chachapoyas, el pepián y las humitas verdes de Tumbes, y el olvido de la tristeza con la chicha de jora, desde la costa hasta el borde de los páramos y las punas, acompañado de moti, tan arraigado en nuestras preferencias saladito y recién tostado, ¡nuestros productos están en la comida local!

La yuca, ya sea frita, sancochada para las tortitas con queso, arrebozadas con huevo o con refrito, un sabor inconfundible lleno de añoranzas de un desayuno familiar que, al incorporar otros alimentos, en su peculiar consumo, mejoran su valor nutricional.

En las comunidades andinas, con desigualdades sociales y de salud de mucho tiempo, Según Ayala (s/f, p. 100), la alimentación ha sido esencialmente en base de vegetales, predominando los tubérculos (papa, oca y mashua), que son ricos en hidratos de carbono, pero pobres en algunos aminoácidos esenciales; sin embargo, el consumo de granos 
(quinua, cañihua y kiwicha), y metionina, y de leguminosas (tarwi, frijol) ha compensado las carencias de los tubérculos. Ambos grupos de alimentos constituyen una importante fuente de energía y nutrientes que permiten una composición más equilibrada de la dieta.

Los tubérculos de las papas dulces se emplean bajo múltiples formas en la alimentación, causas, puré y para la obtención del almidón de papas y los tubérculos de las papas amargas para la elaboración del chuño, moraya, moscko, shile, ckachu-chuño, etc.

La quinoa, de las altas regiones andinas de nuestro Perú, en el siglo XX como quinua, señala Martínez (2014, p. 317), se ha ido redescubriendo gracias a la ciencia que ha ido aportando en forma acumulativa a la nutrición, para combatir el hambre y la malnutrición en los niños y en las mujeres adolescentes prioritariamente.

Se utilizan principalmente, las semillas (granos), previa eliminación del contenido amargo, en la preparación de una gran variedad de comidas y bebidas.

La quinua destaca por ser una buena fuente de proteínas de calidad, fibra dietética, grasas poliinsaturadas y minerales; es importante consumirla como parte de una comida equilibrada junto con otros alimentos a fin de obtener una buena nutrición general.

Entre los granos andinos, la quinua es el de mayor versatilidad para el consumo, pues el grano entero, la harina cruda o tostada, hojuelas, sémola y polvo instantáneo pueden ser preparados en múltiples formas, lo que se traduce en una enorme cantidad de recetas tradicionales como innovadoras (F. Pérez, H. Ikehara, S. Barraza, A. Mortesen, 2016, p. 20).
Es costumbre del Cardenal de Lima ${ }^{1}$, referirse a las semillas de Santa Rosa, al pedir apoyo para la alimentación de los pobres y vulnerables, "frejoles, garbanzos, pallares, quinua, lentejas, y otras más, que ayuden a fortalecer el sistema inmunológico"; el conocimiento desde la ciencia y la práctica de la cultura alimentaria nos demuestra que es posible avanzar para aminorar el impacto de la emergencia sanitaria con nuestros recursos alimentarios, con nuestras preparaciones cotidianas, informando, comunicando o educando en alimentación y nutrición.

\section{Enseñar y aprender de la cultura alimentaria para mejorar la salud}

La pandemia nos ha mostrado que, si se deprimen las respuestas inmunitarias, se incrementa el riesgo de enfermar. Además, con el confinamiento para evitar el contagio, se incrementó tanto la inactividad física como las alteraciones en el estado de ánimo, lo cual, afecta directamente a la salud de la población, especialmente a las personas que padecen diabetes u obesidad.

Si queremos propiciar una mejoría en la salud, la mayor exigencia sería observar comportamientos alimentarios saludables, como son, una alimentación variada, sin excesos, con regularidad, con alimentos frescos para evitar, así, el desarrollo de malos hábitos de alimentación, sumado lo dicho a la evidencia de que los alimentos no trasmiten enfermedades y, las personas sí, para evitar el COVID-19, el lavado frecuente de manos, sobre todo, hacerlo antes de comer o preparar la comida será otro comportamiento exigible.

Solo la Educación hará posible mejorar e influir positivamente los cambios de conducta alimentaria, enseñar para propiciar el proceso de adquisición de hábitos alimentarios saludables

1. Castillo Mattasoglio, Carlos Gustavo (Lima, 28 de febrero de 1950), es un obispo católico peruano, sociólogo, teólogo, Arzobispo de Lima y Primado del Perú desde el 2 de marzo de 2019. 
desde las escuelas, para que la población escolar tome interés y valore nuestras plantas alimenticias.

La información y formación de hábitos, desde la educación, de forma confiable y científica, hará de la alimentación nuestra medicina y nos ayudará a cambiar no solo hacia hábitos más saludables, sino que nos ayudará a volver la mirada al consumo de nuestros recursos, a nuestras preparaciones tradicionales.

Del análisis de los componentes químicos de los alimentos mencionados y aprendidos en la escuela, se reconocerá su calidad hipercalórica e hiperproteica, que ajustados a los requerimientos estimados, según las características de edad, peso, sexo y actividad física, se reconocerán como sumamente útiles a ser incorporados a la dieta diaria y se conseguirá, de esta manera, en base al conocimiento de sus bondades y de su acción, aprendidas en el aula, como una buena alternativa para mejorar la respuesta inmune del organismo y tratar, así, las deficiencias de micronutrientes a partir del consumo de frutas $\mathrm{y}$ verduras, fuentes de vitaminas y minerales, fundamentales para el fortalecimiento del sistema inmune (Academia Española de Nutrición, 2020).
El resguardo sanitario de una alimentación basada en la cultura alimentaria nacional, propicia una gestión preventiva de la pandemia, sumada a las normas de cuidado (uso de mascarillas, distancia en el saludo y una estricta aceptación de reglas colectivas).

\section{CONCLUSIONES}

1. La relación entre el buen comer y la salud desde la Educación es evidente.

2. El COVID-19, mostró las debilidades individuales e institucionales, pero ha despertado una auténtica preocupación por aprender contenidos sobre alimentos $\mathrm{y}$ alimentación.

3. La información, la comunicación y la educación son la estrategia clave para afrontar la situación de la emergencia sanitaria, desde la perspectiva de la alimentación y la nutrición a través de la educación básica regular.

4. Enseñar desde la cultura alimentaria nacional es revalorar la alimentación, para hacer de la educación alimentaria nutricional la mejor estrategia para proteger la salud de todos. 


\section{REFERENCIAS}

Ayala Guido (s/f). Aporte de los cultivos andinos a la nutrición humana. Raíces Andinas. Contribuciones al conocimiento y a la capacitación. I. Aspectos generales y recursos genéticos de las raíces andinas. $\mathrm{p}$. 100.

Brasil.Ministerio de Desarrollo Socialy Combate al Hambre (2012). Marco de Referencia de Educación Alimentaria Nutricional para las Políticas Públicas. Brasilia, DF: MDS. Secretaría Nacional de Seguridad Alimentaria y Nutricional. Recuperado de: https://tinyurl.com/yyvgqljm

Chefs contra el Hambre. Recuperado de: https:// tinyurl.com/y66zpttn

Fondo de las Naciones Unidas para la Agricultura y la Alimentación ( $\mathrm{s} / \mathrm{f}$ ). La Importancia de la Educación Nutricional. Grupo de Educación Nutricional y de Sensibilización del Consumidor de la FAO. Recuperado de: https://tinyurl.com/y2kkjjea

Herrera, F. Plantas alimenticias domesticadas por los inkas, cuyo uso se encuentra poco generalizado. Revista Chaski, Lima, 1941, Volumen 1, Año III, pp. 11-18. Recuperado de: https://tinyurl.com/y22au6ac

Herrera, F. (1943). Plantas Alimenticias y Condimenticias Indígenas del Departamento del Cuzco. Boletín de la Dirección General de Agricultura. Lima, Perú; Año 14, № 4851, pp. 173-195.

Horkheimer, H. (2004). Alimentación y obtención de alimentos en el Perú. Lima: Instituto Nacional de Cultura.

La Iniciativa América Latina y Caribe sin hambre. Recuperado de: http://www.ialcsh. org/

Martínez Enrique, A. (2014). Aspectos Técnicos y Nutricionales. Capítulo No 3.4. En: Bazile D. et al. (Editores), Estado del arte de la quinua en el mundo en 2013: FAO (Santiago de Chile) y CIRAD, (Montpellier, Francia), p. 317. Recuperado de: http://www.fao. org/3/a-i4042s.pdf

Pérez, F., Ikehara, H., Barraza, S., Mortesen, A. (2016). Hupa, Quinua: Semilla Sagrada, Sustento Ancestral. UNESCO, Programa Conjunto Granos Andinos. https://unesdoc. unesco.org/ark:/48223/pf0000261004

Romo-Romo, A., Reyes, C., Janka-Zires, M., Almeda-Valdés, P. (2020). El rol de la nutrición en la enfermedad por coronavirus 2019 (COVID-19). Revista Mexicana de Endocrinología, Metabolismo y Nutrición 7(3):132-143 June 2020. DOI: 10.24875/ RME.20000060

Fecha de presentación: 20-10-20

Fecha de aceptación: 10-11-20 